\title{
EFFECTS OF FILTERS ON DVB-T RECEIVER PERFORMANCE UNDER AWGN, RAYLEIGH, AND RICEAN FADING CHANNELS
}

\author{
AKM Arifuzzaman ${ }^{1}$, Mussab Saleh ${ }^{2}$, and Mohammed Tarique ${ }^{2}$, Rumana Islam ${ }^{1}$ \\ ${ }^{2}$ Department of Electrical and Electronic Engineering, American International University- \\ Bangladesh, Dhaka, Bangladesh \\ arrifuzzaman, rumana@aiub.edu \\ ${ }^{1}$ Department of Electrical Engineering, Ajman University of Science and Technology, \\ Fujairah, United Arab Emirates \\ m.tarique, m.mohammadeajman.ac.ae
}

\begin{abstract}
Digital Video Broadcasting - Terrestrial (DVB-T) has become a very popular technology for terrestrial digital television services. DVB-T is based on Orthogonal Frequency Division Multiplexing (OFDM) technique. OFDM is considered suitable for DVB-T system because of its low Inter-Symbol Interference (ISI). DVB-T has some limitations too including large dynamic signal range and sensitivity to frequency error. To overcome these limitations a good DVB-T receiver is a must. In this paper we address these issues. This paper has two-fold objectives ( $i$ ) to investigate the performances of DVB-T system under different channel conditions, and (ii) to improve performance of DVB-T system by selecting suitable filters in receiver. To investigate the performance of DVB-T system we have considered some popular channel models namely AWGN, Rayleigh, and Ricean. In order to improve the system performance some classic filters like Butterworth, Chebyshev, and elliptic have been included in the receiver. The simulation results show that a careful selection of filter is a must for a DVB-T system. It is also shown that the filter selection should be based on the underlying channel conditions.
\end{abstract}

\section{KEYWORDS}

DVB, DVB-T, multi-carrier, orthogonal, FFT, IFFT, BER, ISI, AWGN, Butterworth, Elliptic, Chebyschev, PSD.

\section{INTRODUCTION}

Since its introduction in 1928 television (TV) has become a cost effective primary source of entertainment and information [1-2]. Originally TV was introduced as an electromechanical system in USA, an all-electronic TV was developed in Europe in the twentieth century [3]. At the same time National Television System Committee (NTSC) was formed in USA. The development of color television system was led by Europe. The Sequential Couleur A Memoire (SECAM) and Phase Alternating Line (PAL) were introduced in Europe. In 1953, NTSC color television was introduced in USA. It remained dominant in the market till the first decade of the $21^{\text {st }}$ century. The first digital television standard (HDTV) was introduced in Japan. Since then digital television has become very popular because of its better quality picture and sound. It uses broadcast spectrum more efficiently. So the television broadcasters could accommodate more channels in a limited spectrum. Until late 1990, digital television broadcasting was costly and it was thought to be impractical. During 1991, broadcasters and equipment manufacturers decided to form a panEuropean platform to develop terrestrial television service. They initiated Digital Video DOI : $10.5121 /$ ijcnc.2013.5408 
Broadcasting (DVB) project [4]. Currently, the DVB project is a consortium of about 250-300 European companies. It has eventually become a worldwide consortium consisting of the members from manufacturers, broadcasters, network operators, software developers, and regulatory bodies. The DVB standard is published by Joint Technical Committee (JTC) of European Telecommunications Standards Institute (ETSI), European Committee for Electrotechnical Standardization (CENELEC), and European Broadcasting Union (EBU). A variety of approaches has been used by DVB to deliver the data services including: (i) Satellite: DVB-S, DVB-S2, DVB-SH, (ii) Cable: DVB-C, DVB-C2, (iii) Terrestrial television: DVB-T, DVB-T2, (iv) Terrestrial television (handhelds): DVB-H, DVB-SH, and (iv) Microwave: DVB-MT,DVBMC, DVB-MS [5]. In this paper, we have considered the DVB-T standard.

The DVB-T system, operating within the existing Very High Frequency Band (50-230 MHz) and Ultra High Frequency Band (470-870 MHz), was introduced in [6]. There are two modes of operations namely " $2 \mathrm{~K}$ Mode" and " $8 \mathrm{~K}$ Mode". The " $2 \mathrm{~K}$ Mode" has been defined for DVB-T transmission and the "8K Mode" has been defined for DVB-H transmission [7]. For limited distance digital video broadcasting the " $2 \mathrm{~K}$ Mode" is considered suitable. On the other hand, for long distance digital video broadcasting the " $8 \mathrm{~K}$ Mode" is preferable. The DVB standard uses different inner code rates. The system also allows two levels of hierarchical channel coding and modulation. The basic functional block diagram of DVB-T standard is shown in Figure 1.

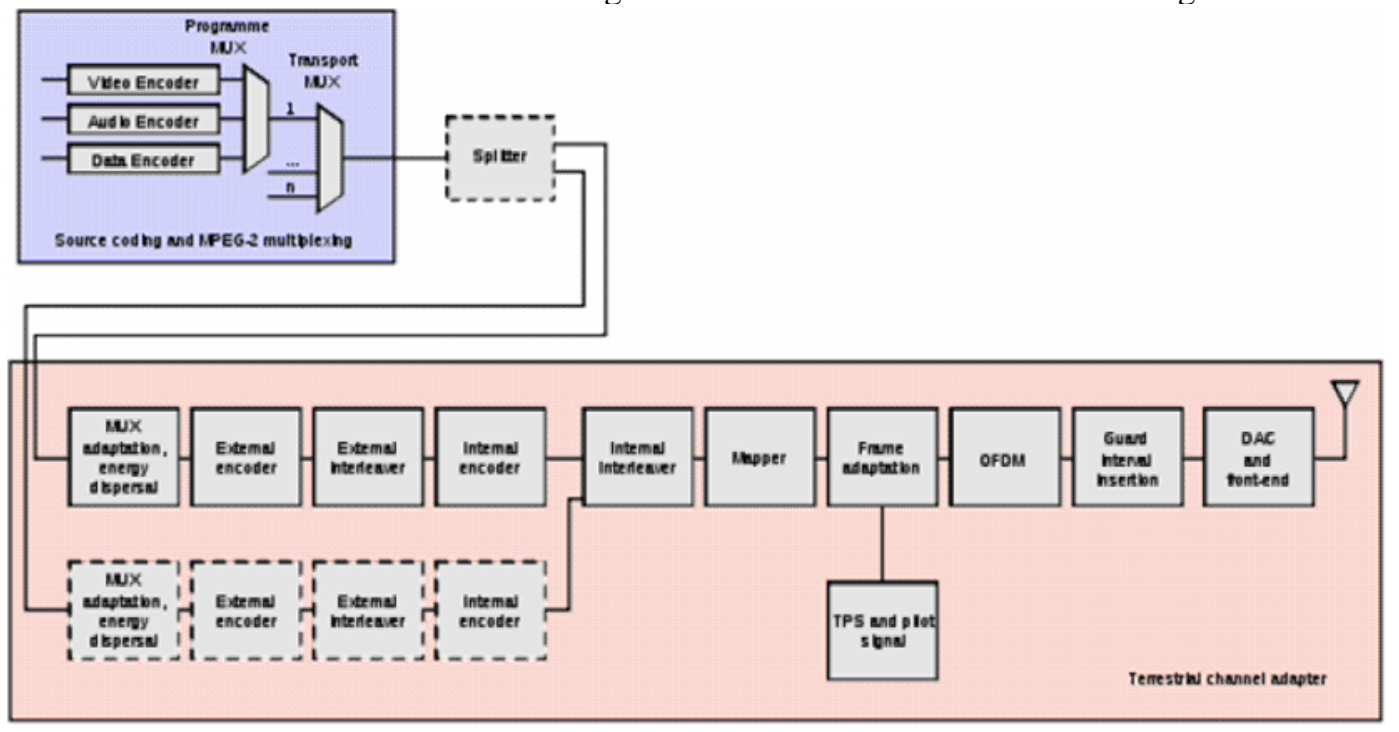

Figure 1. Block diagram of DVB system [1].

Compressed video, compressed audio and data stream encoders are multiplexed into MPEG program streams. Several MPEG program streams are multiplexed into MPEG transport stream. A splitter is used to implement hierarchical transmission. It assists different signals like SDTV and HDTV to be transmitted by using the same carrier. MUX adaptation and energy dispersal convert MPEG transport stream into data packets with proper sequences.

A first level error correction is implemented by using an external coder. A Reed Solomon (RS) code is used for this purpose. In order to cope with the long sequence of errors a convolutional interleaver is used. A second level of error correction is done by using internal encoder. A punctured convolutional coder with the coding rate options of $1 / 2,2 / 3,3 / 4,5 / 6$, and $7 / 8$ is used for this purpose. Another interleaver (see Figure 1) is used to rearrange the data sequence to reduce the effect of burst error. DVB-T has options for different types of modulation namely Quadrature phase Shift Keying (QPSK), 16-QAM, and 64-QAM. A mapper is used to convert the 
International Journal of Computer Networks \& Communications (IJCNC) Vol.5, No.4, July 2013

data symbols into base band complex symbols that are suitable for appropriate modulation. The complex symbols are grouped together to form frame. A DVB-T frame contains 68 symbols. To simplify the reception of DVB-T signal two additional signals are transmitted namely Pilot and TPS signals. Pilot signals are used for the synchronization and equalization. The TPS signal is used for identifying the transmission cell. The sequence of symbols is then modulated by using OFDM technique.

OFDM is a special form of spectrally efficient multicarrier modulation technique that employs densely spaced orthogonal sub-carriers and overlapping spectrums $[6,8]$. The subcarriers are orthogonal with each other and also they can overlap to minimize the bandwidth requirement. OFDM has the ability to reduce or completely eliminate the effect of Inter Symbol Interference (ISI), which arises from the multipath radio wave propagation [9]. Discrete Fourier Transform (DFT) and Inverse Discrete Fourier Transform (IDFT) are used to generate the orthogonal carrier signals. More computationally efficient algorithms like Fast Fourier Transform (FFT) and Inverse Fast Fourier Transform (IFFT) can be used in OFDM. The orthogonal relation among the subcarriers can be jeopardized during the course of transmission of the same through a multipath channel. A multipath channel also introduces Inter Carrier Interference (ICI). Cyclic Prefix (CP) is added with the OFDM symbol to combat ISI and ICI. The CP contains a copy of the last part of the OFDM symbol appended at the front of transmitted OFDM symbol. The length of the CP must be longer than the maximum delay spread of the multipath environment to reduce the ISI. The guard interval insertion component shown in Figure 1 adds the CP with the OFDM symbol. The digital signal produced by the OFDM is transformed into an analog signal by using a Digitalto-Analog Converter (DAC) and it is then modulated by using RF front-end as shown in Figure 1.

\section{RELATED WORKS}

Numerous related works can be found in the literatures. Here, we discussed some of them. The bandwidth efficiency of OFDM systems is achieved by overlapping the orthogonal sub-carriers as mentioned before. Ideally, it is assumed that the waveforms of OFDM transmission have limited band. The spectrum of OFDM symbols overlap only with its adjacent sub-carrier. Practically the OFDM transmission is not band limited. Hence, the spectrums not only interfere with the adjacent carrier, but they also interfere with the other sub-carriers [10]. In order to limit this type of furaway interference, we need to keep the spectrum of the OFDM transmission in a sufficiently low stop-band. Many research works have been done to minimize this type of interference. One of the early solutions is presented in [11]. The authors presented some analysis which led to use of truncated Prolate Spheroidal Wave Function (PSWF) [12]. FIR filters have been designed for transmitter and receiver by using a non-linear programming in [13]. To limit the bandwidth and ISI of OFDM symbol pulse shaping and filtering have been suggested in many works. For example, Raised Cosine Filter (RCF) has been used in [14-17]. The authors have shown in these works that BER can be reduced by using Raised Cosine Filters. The authors show the disadvantages of using reconstruction filters, anti-aliasing filters and other filters in [18]. They proved that these types of filters cause smearing in an OFDM symbol. The authors presented some numerical works and suggested that Chebyshev II filter should be the best choice. This type of filter causes the least smearing. A series of investigations also shows that filtering is an important element to reduce Peak-to-Average Ration (PAR) of OFDM system. The authors suggested in [19] that one should use the clipping method to reduce PAR reduction. It causes both in-band distortion and out-band distortion. Hence, a filter should be used after the clipping to reduce the distortions. A more efficient method for clipping and filtering has been proposed in [20,21]. In these works the authors demonstrated that the clipping and filtering algorithm are better than clipping along to reduce PAPR. A simple method of clipping and filtering has been presented in [22]. Channel estimation is another important element of an OFDM system. The 
channel estimation of an OFDM system by using non-ideal interpolating and decimating filters have been investigated under both AWGN and Rayleigh fading channel in [23]. Two schemes for ICI reduction have been proposed in [24]. An unscented Kalman filter based solution has also been provided in [25]. A high mobility OFDM system has been investigated in this work. The effects of imperfect anti-aliasing filtering have been investigated in [26]. The authors have used a linear phase FIR filter of high order (i.e., $\mathrm{N}=50$ ) in this investigation. In [27] it has been claimed that pulse shaping filter is not good enough for ICI cancellation. A hybrid scheme consisting of pulse shaping and Maximum Likelihood Estimation (MLE) have been combined to cancel ICI in this work.

In all the above mentioned works the investigators focused on a particular filter to improve the performance of OFDM system. Only a few works provided a comparative performance analysis of different types of filter. Moreover, most of the works mentioned above use one type of channel condition. In this work we considered different channel conditions namely AWGN, Rayleigh channel, and Ricean channels. The performance of DVB-T system has been investigated in this work under different channel conditions. In addition to this, some classic filters namely Butterworth, Elliptic, and Chebyshev filters have been used in the receiver of DVB-T system. We varied some filter parameters like cut-off frequency and filter order to investigate the performance of DVB-T receiver under different channel conditions.

\section{DVB FRAME STRUCTURES}

Each DVB-T symbol is transmitted in the form of a frame. Each frame consists of 68 OFDM symbols [5]. Each symbol consists of a number of sub-carriers. There are 1705 sub-carriers and 6817 sub-carriers in the " $2 \mathrm{~K}$ Mode" and " $8 \mathrm{~K}$ Mode" respectively. The symbol interval has two components namely a useful part and a guard interval. The guard interval carries cyclic prefix (CP) and it is inserted at the front of the useful component as shown in Figure 2. Here, $T_{F}$ is the frame duration, $\mathrm{T}_{\mathrm{U}}$ is the duration of useful data, $\mathrm{T}_{\mathrm{G}}$ is the guard interval, and $\mathrm{T}_{\mathrm{S}}$ is the duration of a symbol. Four guard intervals have been defined in DVB standard namely 1/4, 1/8, 1/16 and 1/32. The symbols of a DVB-T frame are numbered from 0 to 67. In DVB standard all symbols contain data and reference information. The carriers are indexed from $K_{\min }$ to $K_{\max }$, where $K_{\min }=0$ and $\mathrm{K}_{\max }=1704$ for " $2 \mathrm{~K}$ Mode" and $\mathrm{K}_{\min }=0$ and $\mathrm{K}_{\max }=6816$ for " $8 \mathrm{~K}$ Mode". The spacing between $K_{\min }$ and $K_{\max }$ is defined by $(\mathrm{K}-1) / \mathrm{T}_{\mathrm{U}}$, where the spacing between adjacent carriers is $1 / \mathrm{T}_{\mathrm{U}}$, and $\mathrm{K}$ is the number of the sub-carriers. Some other OFDM parameters for the " $2 \mathrm{~K}$ Mode" and " $8 \mathrm{~K}$ Mode" for $8 \mathrm{MHz}$ channel are listed in Table 2 and Table 3.

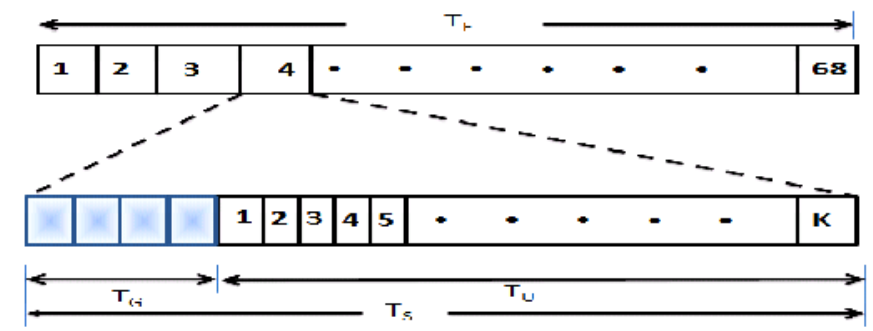

Figure 2: OFDM frame and symbol structure.

The transmitted OFDM symbol can be mathematically expressed as

$$
s(t)=\operatorname{Re}\left\{e^{2 \pi f_{c} t} \sum_{m=0}^{\infty} \sum_{l=0}^{67} \sum_{k=K_{\text {min }}}^{K_{\max }} c_{m, l, k} \times \psi_{m, l, k}(t)\right\}
$$


, where

$$
\begin{aligned}
& \psi_{m, l, k}(t)=\left\{\begin{array}{l}
e^{j 2 \pi \frac{k^{\prime}}{T_{U}}\left(t-T_{G}-l \times T_{S}-68 \times m \times T_{S}\right.} \quad(l+68 \times m) \times T_{S} \leq t \leq(l+68 \times m+1)
\end{array}\right. \\
& \psi_{m, l, k}(t)=0, \text { Otherwise }
\end{aligned}
$$

, where $k=$ carrier number, $l=$ OFDM symbol number, $m=$ the frame number, $K=$ the number of transmitted sub-carrier, $f_{c}=$ the central frequency, $k \square=$ the carrier index related to the center frequency, and $\mathrm{c}_{\mathrm{m}, 1, \mathrm{k}}=$ complex symbol for carrier $k$ of the data symbol number $l$ in frame $m$. The $\mathrm{c}_{\mathrm{m}, \mathrm{l}, \mathrm{k}}$ values are normalized according to the constellation points of the modulation alphabet used.

Table 2. OFDM Parameters for " $2 \mathrm{~K}$ " and " $8 \mathrm{~K}$ " mode

\begin{tabular}{|l|l|l|}
\hline Parameter & $8 \mathrm{~K}$ mode & $2 \mathrm{~K}$ mode \\
\hline Number of carriers $\mathrm{K}$ & 6817 & 1705 \\
\hline Value of carrier number $\mathrm{K}_{\min }$ & 0 & 0 \\
\hline Value of carrier number $\mathrm{K}_{\max }$ & 6816 & 1704 \\
\hline Duration $\mathrm{T}_{\mathrm{U}}$ & $896 \mathrm{~s}$ & $224 \mathrm{~s}$ \\
\hline Carrier spacing $1 / \mathrm{T}_{\mathrm{U}}$ & $1116 \mathrm{~Hz}$ & $4464 \mathrm{~Hz}$ \\
\hline Spacing between $\mathrm{K}_{\min }$ and $\mathrm{K}_{\max }(\mathrm{K}-1) / \mathrm{T}_{\mathrm{U}}$ & $761 \mathrm{MHz}$ & $761 \mathrm{MHz}$ \\
\hline
\end{tabular}

\begin{tabular}{|c|c|c|c|c|c|c|c|c|}
\hline Mode & \multicolumn{4}{|r|}{$8 \mathrm{~K}$ mode } & \multicolumn{4}{|r|}{$2 \mathrm{~K}$ mode } \\
\hline $\begin{array}{r}\text { Guard } \\
\text { interval } \\
\mathrm{T}_{\mathrm{G}} / \mathrm{T}_{\mathrm{U}}\end{array}$ & $1 / 4$ & $1 / 8$ & $1 / 16$ & $1 / 32$ & $1 / 4$ & $1 / 8$ & $1 / 16$ & $1 / 32$ \\
\hline $\begin{array}{r}\text { Duration of } \\
\text { symbol part } \\
\mathrm{T}_{\mathrm{U}}\end{array}$ & & & & $\begin{array}{l}8192 \times \mathrm{T} \\
=896 \mathrm{~s}\end{array}$ & & & & $\begin{array}{l}2048 \times \mathrm{T} \\
=224 \mathrm{~s}\end{array}$ \\
\hline $\begin{array}{r}\text { Duration of } \\
\text { Guard } \\
\text { Interval } \mathrm{T}_{\mathrm{G}} \\
\end{array}$ & $\begin{array}{l}2048 \times \mathrm{T} \\
=224 \mathrm{~s}\end{array}$ & $\begin{array}{l}1024 \times \mathrm{T} \\
=112 \mathrm{~s}\end{array}$ & $\begin{array}{l}512 \times \mathrm{T} \\
=56 \mathrm{~s}\end{array}$ & $\begin{array}{l}256 \times \mathrm{T} \\
=28 \mathrm{~s}\end{array}$ & $\begin{array}{l}512 \times \mathrm{T} \\
=56 \mathrm{~s}\end{array}$ & $\begin{array}{l}256 \times \mathrm{T} \\
=28 \mathrm{~s}\end{array}$ & $\begin{array}{l}128 \times \mathrm{T} \\
=14 \mathrm{~s}\end{array}$ & $\begin{array}{l}64 \times \mathrm{T} \\
=7 \mathrm{~s}\end{array}$ \\
\hline $\begin{array}{r}\text { Symbol } \\
\text { Duration } T_{S}\end{array}$ & $\begin{array}{l}10240 \times \mathrm{T} \\
=1120 \mathrm{~s}\end{array}$ & $\begin{array}{l}9216 \times \mathrm{T}= \\
1008 \mathrm{~s}\end{array}$ & $\begin{array}{l}8704 \times \mathrm{T}= \\
952 \mathrm{~s}\end{array}$ & $\begin{array}{c}8448 \times \mathrm{T} \\
924 \mathrm{~s}\end{array}$ & $\begin{array}{l}2560 \times \mathrm{T} \\
=280 \mathrm{~s}\end{array}$ & $\begin{array}{c}2304 \times \mathrm{T} \\
252 \mathrm{~s}\end{array}$ & $\begin{array}{l}2176 \times \mathrm{T} \\
=238 \mathrm{~s}\end{array}$ & $\begin{array}{r}2112 \times \mathrm{T} \\
231 \mathrm{~s}\end{array}$ \\
\hline
\end{tabular}

Table 3. Additional Parameter

\section{DVB TRANSMITTER AND RECEIVER MODELS}

The transmitter and the receiver models proposed in [28] have been used in this investigation. Figure 3(a) and Figure 3(b) show the basic signal processing steps done in the transmitter and the receiver of DVB-T system. The number of sub-carriers in DVB-T system is 1705 . Hence, we considered 1705 4-QAM symbols as the input data to the transmitter model. The bandwidth and the carrier frequency are $8.0 \mathrm{MHz}$ and $90 \mathrm{MHz}$ respectively. For the OFDM symbol generation we chose 4096-point IFFT. The output carriers of IFFT are then converted into continuous time signal. A pulse shaping filter has been used for this purpose. This is low pass filter [29] denoted by Digital-to-Analog (D/A) filter in the block diagram of Figure 3(a). In the next step a D/A filter with a sharp bandwidth is used. We choose a Butterworth filter of order 13 for this purpose. Finally, the carrier modulation is performed at the last stage.

Designing an OFDM receiver has been an active research topic for the last few years. Most of the research works conducted has been focused on OFDM receiver design. The OFDM receiver 
model used in this investigation is shown in Figure 3(b). The OFDM receiver performs the inverse operations that were done in the transmitter. First, the demodulation is done. Then a lowpass filter is used to recover the continuous version of the OFDM symbol. The delays produced by the reconstruction and demodulation filters are then compensated. The resultant signal is then sampled to convert continuous OFDM symbol into its discrete version. The resulted signal is then passed through a 4096-FFT. Finally a QAM estimator has been used to recover the original data.

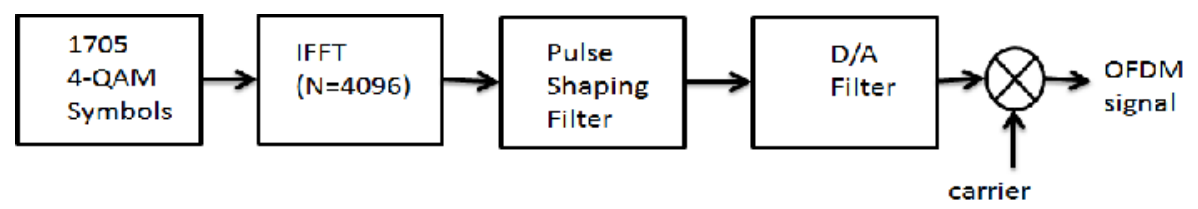

(a) Transmitter

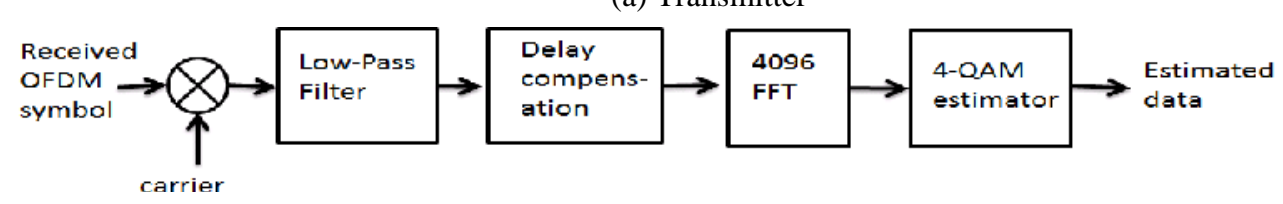

(b) Receiver

Figure 3. OFDM Transmitter and Receiver model.

Finally, the resultant data is estimated by using a 4-QAM estimator. Several investigations show that a significant level of OFDM spectrum falls outside the nominal bandwidth. Hence an appropriate filter should be selected in the receiver to minimize this out of band spectrum. In this investigation three classic filters namely Butterworth, Chebyshev, and elliptic filters have been considered.

\section{FiLTER SELECTION}

Among all these three classic filters the Butterworth filter has drawn considerable attention in communication system design. This type of filter exhibits a flat pass band. The roll-off of Butterworth filter is very smooth and monotonic. To generate the spectrum of different filters we used MATLAB built in functions [30-33]. The spectrums of Butterworth, Chebyshev, and elliptic filter of the same order (i.e., 3) and the cut-off frequency of $200 \mathrm{MHz}$ are shown in Figure 4. For Chebyshev filter the pass band ripple was set to $10 \mathrm{~dB}$ (peak-to-peak). In elliptic filter the pass band ripple was set to $0.5 \mathrm{~dB}$ and the stop band ripple was set to $10 \mathrm{~dB}$. Compared to Butterworth filter the Chebyshev filter response has a faster roll-off. But, it allows ripple in the pass band as shown in the Figure 4. There is a trade-off between the ripple and the roll-off characteristic of this type of filter. As the ripple increases, the roll-off becomes sharper. The Chebyshev filters are classified as Type-I and Type-II. Type-I Chebyshev filter has ripple in the pass band. On the other hand Type-II Chebyshev filter has ripple in the stop band. Since Type-II Chebyshev filter is rarely used we consider only Type-I Chebyshev filter in this investigation. The sharp transition between the pass band and the stop band of a Chebyshev filter produces smaller absolute errors and faster execution speed than a Butterworth filter. The cut-off slope of an elliptic filter is steeper that of a Butterworth and Chebyshev filters. But, this type of filter has ripple in the stop band. Compared with the same order Butterworth and Chebyshev filters, the elliptic filters provide the sharpest transition between the pass band and the stop band. For this reason the elliptic filters have wide applications. Based on the spectrum characteristics of Butterworth, Chebyshev, and Elliptic filters we have selected Butterworth 
filter for the rest of this investigation. A comparative performance analysis of Butterworth filter with other two filters has been presented at the end of this paper.
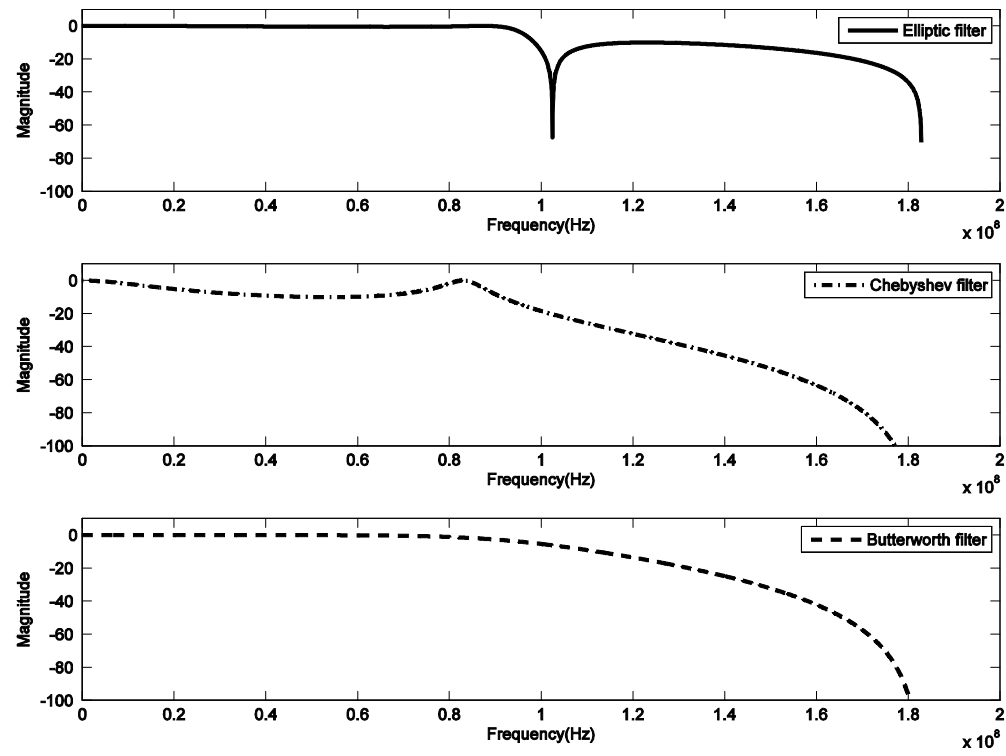

Figure 4. The spectrums elliptic, Chebyshev, and Butterworth filters.

\section{AWGN, RAYLEIGH FADING, AND RICEAN CHANNEL MODELS}

In this paper we have investigated the performances of DVB-T system under different channel conditions namely Additive White Gaussian Noise (AWGN), Rayleigh channel, and Ricean channel. Among these channel models the simplest one is the AWGN channel. This channel model has been commonly used in communication system design. In this model the impairment to communication is modeled by a linear addition of white noise with a constant power spectral density. The probability density function (pdf) of noise is modeled by a Gaussian distributed random distribution. This Gaussian noise comes from many natural sources such as the thermal vibrations of atoms in conductors, shot noise, black body radiation from the earth and other warm objects, and from celestial sources such as the Sun. The AWGN channel is also a good model model for many communication links such as satellite link and deep space communication links. 


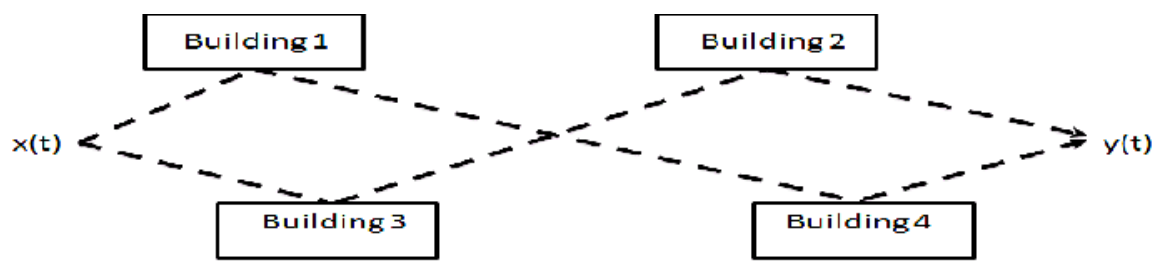

Figure 5. Multipath propagation in wireless channel.

The disadvantage of AWGN model is that it is not applicable to model a real world terrestrial link. Because AWGN channel model does not consider some important characteristics including multipath, terrain blocking, and interference of a terrestrial link. Rayleigh fading channel model is another commonly used model that takes account of the variation of signal under multipath condition. A multipath propagation scenario is depicted in Figure 5. In this scenario the transmitted signal $\mathrm{x}(\mathrm{t})$ travels a number of paths from a transmitter to a receiver. If the transmitted signal is expressed as $x(t)=A \cos \left(2 \pi f_{c} t+\phi\right)$, the received signal can be expressed as

$$
\begin{aligned}
r(t) & =\sum_{n=1}^{L} A_{i} \cos \left(2 \pi f_{c} t+\phi_{i}\right) \\
& =\cos 2 \pi f_{c} t \sum_{n=1}^{L} A_{i} \cos \left(\phi_{i}\right)-\sin 2 \pi f_{c} \sum_{n=1}^{L} A_{i} \sin \left(\phi_{i}\right)
\end{aligned}
$$

,where $A_{i}$ is the amplitude of the $i$ th path, $\phi_{i}$ is the phase of the $i$ th path, $L$ is the number of paths between the transmitter and the receiver. If the reflective objects (i.e., buildings) are assumed to be uniformly located in the propagation path, the variables $X=\sum_{n=1}^{L} A_{i} \cos \left(\phi_{i}\right)$ and $X=\sum_{n=1}^{L} A_{i} \sin \left(\phi_{i}\right)$ become Gaussian random variables by Virtue of Central Limit theorem. Hence, the received signal amplitude $|r(t)|=\sqrt{X^{2}+Y^{2}}$ becomes a Rayleigh distributed random variables defined by probability density function (pdf) given by follows:

$$
\begin{aligned}
p(r) & =\frac{r}{\delta^{2}} \exp \left(-\frac{r^{2}}{2 \delta^{2}}\right) \quad r \geq 0 \\
& =0 \quad, \text { Otherwise }
\end{aligned}
$$

,where $\delta^{2}$ is the variance of the signal. 


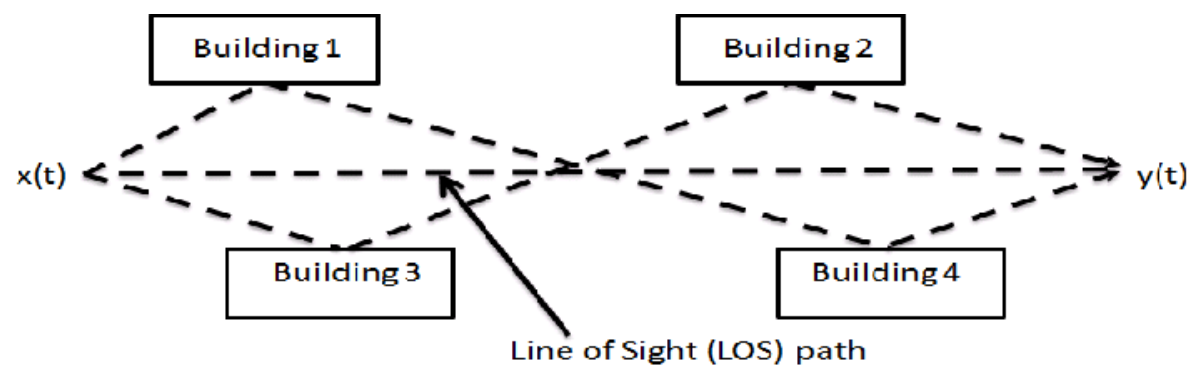

Figure 6. Multipath propagation in wireless channel with a dominant LOS path.

The signal from a transmitter may be reflected by a number of objects (i.e., multipath propagation) in addition to a dominant Line of Sight (LOS) path as shown in Figure 6. At the receiver the LOS path has an effect of adding a DC component with the multipath component. The addition of a strong LOS signal with a number of multiple paths resulted in a Ricean distribution. The probability density function (pdf) of Ricean distribution is expressed as

$$
\begin{aligned}
p(r) & =\frac{r}{\delta^{2}} \exp \left(-\frac{r^{2}+A^{2}}{2 \delta^{2}}\right) I_{0}\left(\frac{A r}{\delta^{2}}\right) \quad A \geq 0, r \geq 0 \\
& =0 \text {, Otherwise }
\end{aligned}
$$

, where parameter $A$ denotes the maximum amplitude of the dominant signal and $\mathrm{I}_{0}(\bullet)$ is the modified Bessel function of the first-kind of zero order. The Ricean distribution is often described in terms of $K$ parameter defined by $K=\frac{A^{2}}{2 \delta^{2}}$. In terms of $\mathrm{dB}$ the $\mathrm{K}$ is defined as

$$
K(d B)=10 \log \frac{A^{2}}{2 \delta^{2}}
$$

As $\mathrm{A} \rightarrow \infty, \mathrm{K} \rightarrow 0$, the Ricean distribution becomes Rayleigh distribution.

\section{DVB TRANSMISSION UNDER AWGN, RAYLEIGH AND RICEAN CHANNELS}

In order to investigate the performance of DVB-T transmission under different channel conditions we conducted simulations in MATLAB. We selected DVB-T with " $2 \mathrm{~K}$ Mode". The simulation parameters were set according to those listed in Table 1 and Table 2 as mentioned in an earlier section. The simulation results in terms of Symbol Error Rate (SER) are shown in Figure 7. This figure shows that the SER decreases with the increase in signal-to-noise (SNR) ratio. It is also shown that for a given SNR, DVB-T has the minimum SER for AWGN channel. But, the multipath effects result in higher SER in Rayleigh fading channel and Ricean fading channel (with $\mathrm{K}=25 \mathrm{~dB}$ ) environment. The SER is low in Ricean channel compared to that of Rayleigh fading channel because of its strong LOS path component. 


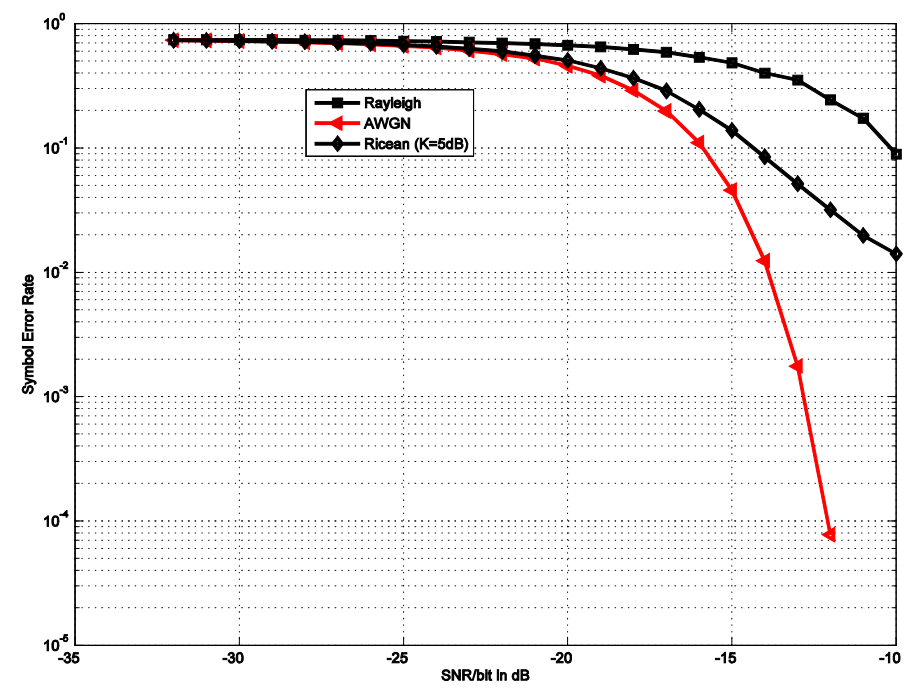

Figure 7. The SER rate performances under AWGN, Rayleigh, and Ricean channel.

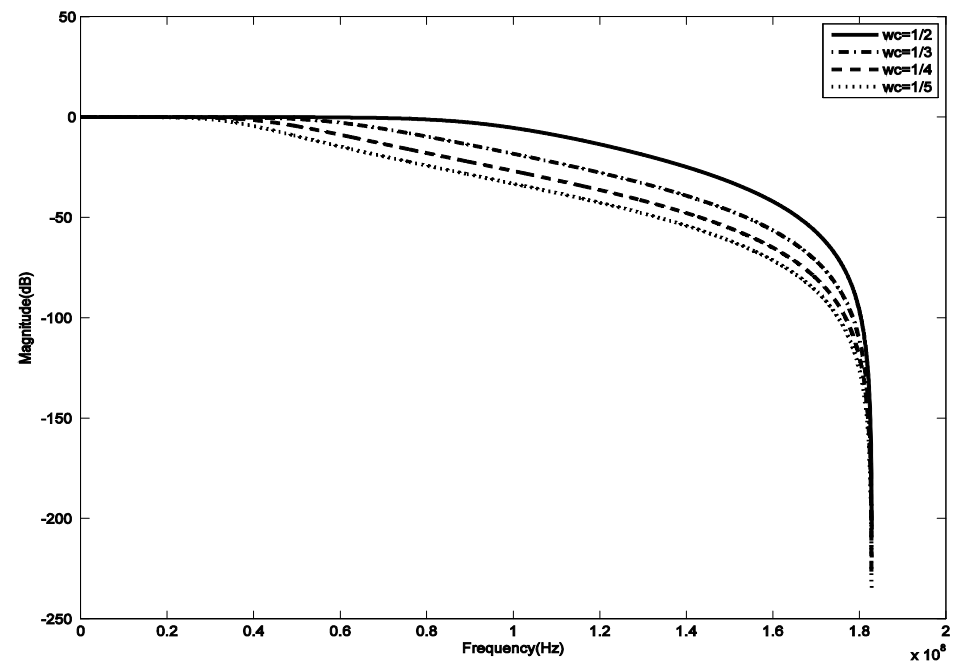

Figure 8. Magnitude spectrum of Butterworth filter.

To investigate the performances of DVB system we choose the simplest AWGN channel model and we choose a Butterworth filter. The frequency domain characteristic of the Butterworth filter depends on two important parameters namely (i) the order of filter, and (ii) the cut-off frequency. Since the OFDM based DVB-T system suffers from frequency error we maintained same filter order (i.e., $\mathrm{N}=3$ ), but we varied the cut-off frequency. The magnitude spectrums of Butterworth filter with varying cut-off frequency are shown in Figure 8. It is depicted in this figure that the magnitude spectrum attenuates sharply as the cut-off frequency is reduced. Higher cut-off frequencies cause higher attenuation rate in this type of filter. The cut-off frequencies are chosen arbitrarily denoted by $\mathrm{w}_{c}=1 / 2,1 / 3,1 / 4$ and $1 / 5$. The cut-off frequency labeled as $\mathrm{w}_{\mathrm{c}}=1 / 2$ represents 
the highest cut-off frequency and the cut-off frequency labeld as $\mathrm{w}_{\mathrm{c}}=1 / 5$ represents the lowest cutoff frequency.

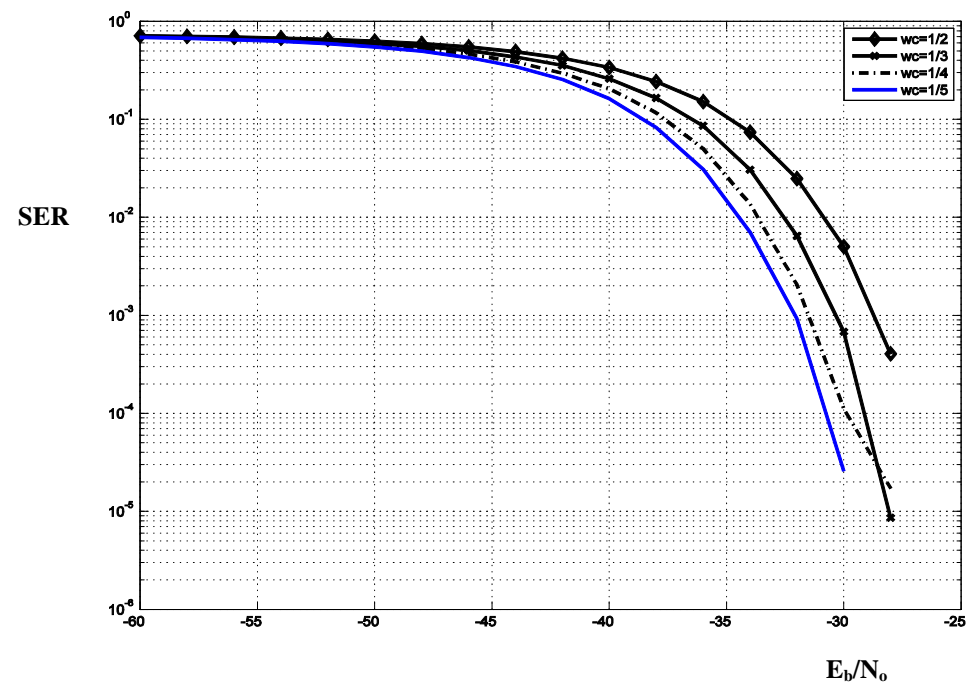

Figure 9. The SER rate performances under AWGN channel with different cut-off frequency.

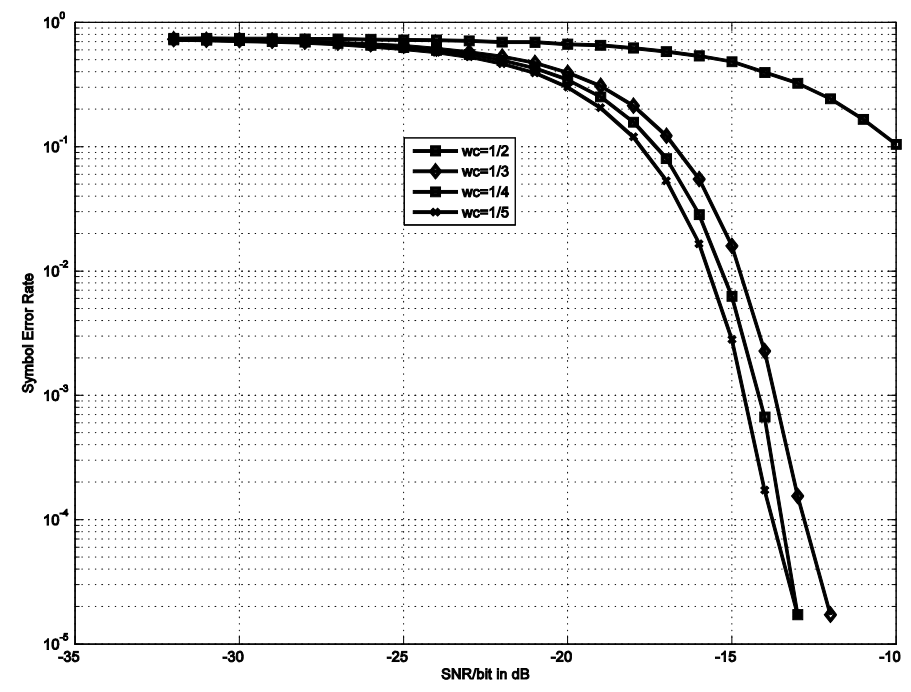

Figure 10. The SER performances of DVB-T under Rayleigh channel.

The SER performances of DVB system under different channel conditions namely AWGN, Rayleigh, and Ricean fading channels are shown in Figure 9, Figure 10, and Figure 11 respectively. In all these simulations we used Butterworth filter and we varied the filter cut-off frequencies. Figure 9 shows that the SER performance of DVB-T system can be improved by decreasing the cut-off frequency under AWGN channel condition. It also shows that SER can be decreased by almost 10 times on an average if a tighter cut-off frequency is used. The effects of filters are more evident for Rayleigh fading channel as shown in Figure 10. Since the Rayleigh fading channel causes the signal to fade significantly due to multipath effects, the filter cut-off frequency selection is more important in such channel environment. It is depicted in the same 
figure that the SER can be significantly reduced if the filter cut-off frequency is changed from $\mathrm{w}_{\mathrm{c}}=1 / 2$ to $\mathrm{w}_{\mathrm{c}}=1 / 5$. The effects of filter on DVB performance under Ricean fading condition is shown in Figure 11. This figure shows that a moderate improvement in SER can be achieved by varying the filter cut-off frequency. This SER improvement is not significant like that of Rayleigh fading case. But, this improvement in SER is better than that of AWGN case.

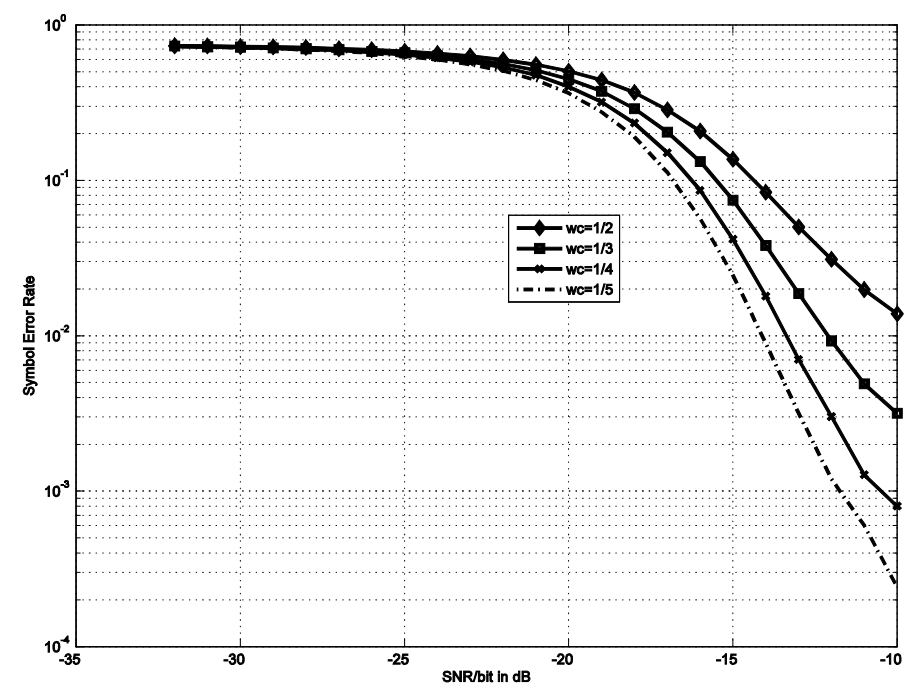

Figure 11. Comparison of filter performance under Ricean Channel.

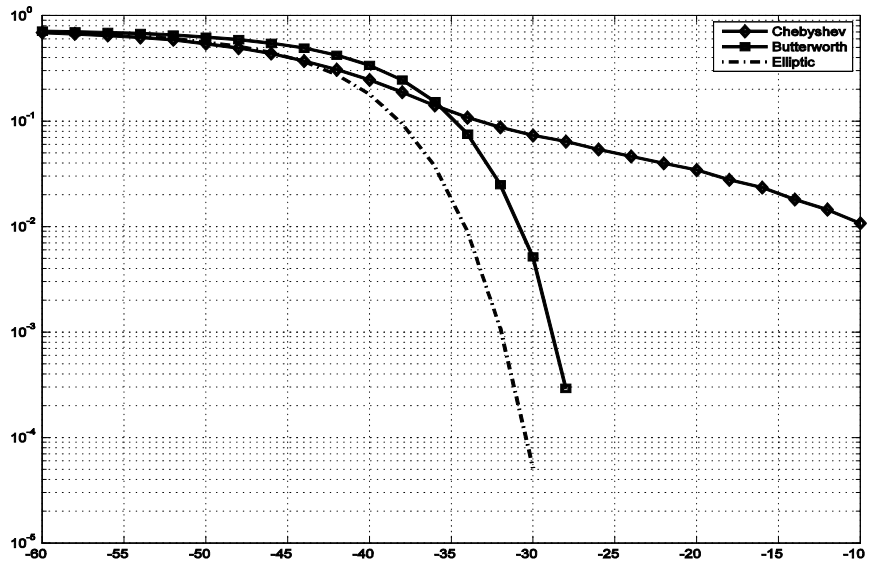

Figure 12. Comparison of different filters performance under AWGN Channel.

\section{COMPARISON OF DIFFERENT FILTERS}

In the DVB signal reception Butterworth filter has drawn considerable attention. In this section we present some results which show a performance comparison of DVB system by using some other popular filters like Chebyshev and elliptic filter. These filters have been tested also under different channel conditions here. For fair comparison we used Butterworth, Chebysheb, and Elliptic filters of the same order and the same cut-off frequencies. Only the pass band ripple has been set to $10 \mathrm{~dB}$ in Chebyshev filter. In elliptic filter both pass band and stop band ripple have been chosen $10 \mathrm{~dB}$ and $0.5 \mathrm{~dB}$ respectively. The simulation results are shown in Figure 12, 
Figure 13, and Figure 14. The SER performances of these three filters under AWGN channel is shown in Figure 12. It shows that an elliptic filter is a good candidate for DVB signal reception under AWGN channel. The Chebyshev filter performs poor in this type of channel. The Butterworth filter shows a fair performance. The results for Rayleigh fading channel are presented in Figure 13. It is shown therein that the Chebyshev filter is good candidate for Rayleigh fading channel. Although an elliptic filter is the best candidate for AWGN channel condition, it performs poor under Rayleigh fading channel condition. Finally, Figure 14 shows that the Butterworth filter is the best candidate for Ricean channel condition. But, the performances of the elliptic and Chebyshev filters are almost similar.

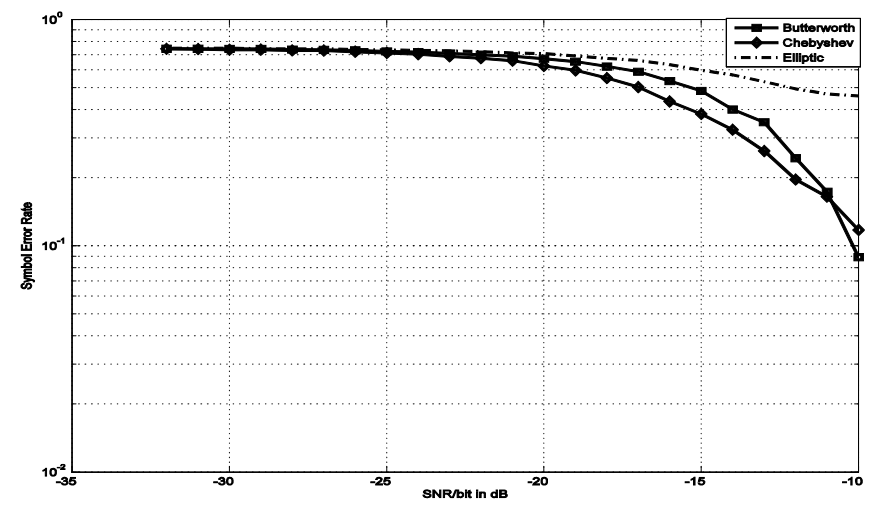

Figure 13. Comparison of filter performance under Rayleigh Channel.

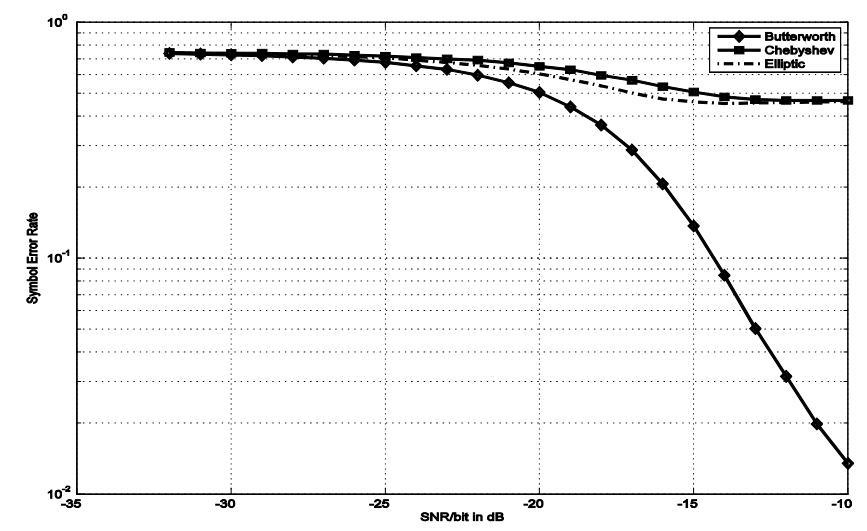

Figure 14. Comparison of filter performance under Ricean channel.

\section{Conclusions}

In this paper, the performance of DVB receiver has been investigated under different channel condition. It is shown that the performances of DVB system depends on the underlying channel conditions. It is also shown that a suitable filter is essential for DVB receiver. The simulation results show the DVB-T receiver performance is very sensitive to the filters. It is also shown a tighter cut-off frequency is required in order to reduce SER. Several filters have been proposed 
International Journal of Computer Networks \& Communications (IJCNC) Vol.5, No.4, July 2013

for DVB receiver. Among these filters, the Butterworth filter is the most popular choice. In this paper, we investigated the performances of DVB receiver by using Butterworth filters of different cut-off frequencies. It is shown in this paper that a Butterworth filter may not be suitable for all channel conditions. In some cases other type of filters like Chebyshev and elliptic filters can perform better than Butterworth filter. Hence, a careful filter selection is very important for a DVB receiver. The selection of filter should be based on channel condition. But, in all cases a tighter cut-off frequency is desirable.

\section{REFERENCES}

[1] Wu, Y., Hirakawa S., Reimers, U.H., and Whitakaer, J., “ Overview of Digital Television Development Worldwide”, In the Proceedings of IEEE, Vol. 94, January 2006, pp. 8-21

[2] Udelson, J.H., " The Great Television Race: A History of the American Television Industry”, University of Alabama Press, 1982

[3] "Television History" available at http://www.tvhostory.tv

[4] Www.dvb.org

[5] DVB Project, Introduction to the DVB Project, DVB Fact Sheet, March 2007

[6] ETSI EN 300 744, Digital Video Broadcasting (DVB): Measurement Guidelines for DVB system, v. 1.2.1, May 2001.

[7] Popovic, M.L.; Sunjevaric, M.M.; Vujasinovic, Z.J. , "Effects of DVB-H and 3G cellular networks integration ", Proceedings of the $10^{\text {th }}$ International Conference on Telecommunications in Modern Satellite cable and Broadcasting Services, Serbia, October 2011, pp. 641-644

[8] R. V. Nee and R. Prasad, OFDM Wireless Multimedia Communications, Norwood, MA: Artech House, 2000.

[9] Man-On Pun, Michele Morelli, and C-C Jay Kuo, "Multi-Carrier Techniques for Broadband Wireless Communications: A Signal Processing Perspective,”, Imperial College Press, 57 Shelton Street, Covent Garden, London WC2H 9HE.

[10] Hirosaki, B. "An Analysis of automatic equalizer for orthogonally multiplexed QAM system", IEEE Transaction on Communication, Vol. com 28, No. 1, 1980, pp. 73-83

[11] Vahlin, N., and Holte, N., " Optimal finite duration pulses for OFDM", IEEE Transaction on Communications, Vol. 44, No. 11, January 1996, pp. 10-14

[12] Slepian, D. and Pollak,H., “ Prolate speroidal wave functions , Fourier analysis and uncertaintyI”, Bell System Technical Journal, Vol. 40, January 1961, pp. 43-63

[13] Chen,H.H., and CAI, X.D., " Optimization of Transmitter and Receiver Filters for OQAM-OFDM Systems using Non-linear Programming”, IEEE Transaction on Communications, Vol. E80-B, No. 11, November 1997, pp. 1680-1687

[14] Aldis, J.P., Althoff, M.P. and Van-Nee, R., " Physical layer architecture and performance in the WAND user trial system”, In the proceedings of ACTS Mobile Conference, Grenada, Spain, 1996, pp. 196-203

[15] Gudmundson, M. and Anderson, P.O., “Adjacent channel interference in an OFDM system”, In the Proceedings of IEEE Vehicular Technology Conference, 1996, pp. 918-922

[16] Cimini, L.J., "Analysis and simulation of a digital mobile channel using orthogonal frequency division multiplexing”, IEEE Transaction on Communications, Vol. 33, 1985, pp. 665-675

[17] Khalid Aslam, Bodiuzzaman,M., Uddin J., and Kulesza, W, "Using Raised Cosine Filter to Reduce Inter- Symbol Interference in OFDM with BPSK technique", Computer Science Journal, Vol. 1, No. 2, August 2011, pp. 115-119

[18] Faulkner, M., " The effects of Filtering on the performance of OFDM systems", IEEE Transactions on Vehicular Technology, Vol. 49, No.5, September 2000, pp. 1877-1884

[19] Prafullah D. G. and Siddharth A. L., " PAPR Performance of OFDM system by using Clipping and Filtering”, International Journal of Advances in Engineering and Technology, Vol. 6, No. 2, May 2013, pp. 789-794

[20] Wang , L. and Tallanbara, C., "A simplified clipping and Filtering technique for PAR Reduction in OFDM systems”, IEEE Signal Processing Letters, Vol. 12, No. 6, June 2005, pp. 453-456

[21] Zhong, X., Qi. J, and Bao, J., " Using clipping and filtering algorithm to reduce PAPR of OFDM system", In the Proceedings of the International Conference on Electronics, Communication, and Control, September 2011, Ningbo, China, pp. 1763-1766 
International Journal of Computer Networks \& Communications (IJCNC) Vol.5, No.4, July 2013

[22] Aziz W., Ahmed E., Abbas G., Saleem, S., and Islam, Q. " PAPR Reduction in OFDM using Clipping and Filtering", World Applied Sciences Journal, January 2012, Vol. 11, pp. 1495-1500

[23] Ho C.K., Sun S., and Farhang B., “ Detrimental Effects of filtering in an OFDM system using pilot based channel estimation", In the Proceedings of the $13^{\text {th }}$ IEEE International Symposium on Personal, Indoor, and Mobile Radio Communication, September, 2002, Vol. 3, pp. 1316-1320

[24] Naveen V,.J., and Rajeswan K.R, “ ICI Reduction using Extended Kalman Filter in OFDM system”, International Journal of Computer Application, Vol. 17, No. 7, March 2011, pp. 15-22

[25] Li X., and Cimini L.J, "Effects of clipping and filtering on the performance of OFDM", IEEE Communication Letter, Vol. 2, No.5, May 1998, pp. 131-138

[26] Klinler, F. and Scelze, H., “ Adjacent Channel Interference with Imperfect Anti-Aliasing Filtering”, In the Proceedings of the $12^{\text {th }}$ International OFDM- Workshop , August 07, Hamburg, Germany

[27] Saritha , H.M. and Kulakarni, M. , " A novel scheme using MLE with pulse shaping for ICI cancellation in OFDM system”, In the Proceedings of International Conference on Communication and Informatics, January 2012, Coimbatore, India, pp. 1-5.

[28] OFDM Simulation using Matlab available at http://www.ece.gatech.edu/research/ labs/ sarl/ tutorials / OFDM/Tutorial_web.pdf

[29] John G. Proakis and Dimitris G. Manolakis, "Digital Signal Processing : Principles, Algorithms, and Applications", Fourth Edition, Prentice Hall, Upper Saddle River, New Jersey, USA

[30] Vinay K. Ingle and John G. Proakis, "Digital Signal Processing using MATLAB”, Thomson Bookware Companion Series

[31] Andreas Antoniou, “ Digital Signal Processing: Signals, Systems and Filters”, McGraw Hill, New York, USA

[32] Sanjit K Mitra, “ Digital Signal Processing: A Computer Base Approach”, McGraw Hill, New York, USA

[33] Tamal Bose, “Digital Signal and Image Prcoessing”, John Wiley and Sons, New Jersey, USA 